Proc. Indian Acad. Sci. (Chem. Sci.), Vol. 96, No. 5, March 1986, pp. 321-326.

(C) Printed in India.

\title{
Polychalcone derived from 8-acetoxyquinoline-5-aldehyde
}

\author{
H S PATEL \\ Department of Chemistry, Sardar Patel University, Vallabh Vidyanagar 388 120, India \\ MS received 24 August 1985; revised 23 December 1985

\begin{abstract}
Fries rearrangement of 8-acetoxyquinoline-5-aldehyde (AQA) in nitrobenzene using $\mathrm{AlCl}_{3}$ as catalyst affords the polychalcone poly (8-hydroxy-5,7-quinolinylenecarbonylvinylene) (PHQCV). The PHQCV samples were characterized by elemental analysis, IR spoctroscopy, molecular weight, intrinsic viscosity and TGA.

Polymeric metal chelates of $\mathrm{Cu}^{2+}, \mathrm{Fe}^{3+}, \mathrm{Co}^{2+}, \mathrm{Ni}^{2+}, \mathrm{Mn}^{2+}, \mathrm{Zn}^{2+}$ and $\mathrm{UO}_{2}^{2+}$ with PHQCV polychalcone were prepared and characterized.
\end{abstract}

Keywords. 8-Acetoxyquinoline-5-aldehyde; polychalcone; poly(8-hydroxy-5,7-quinolinylenecarbonylvinylene); polychelates of polychalcone; poly(8-hydroxy-5,7-quinolinylenecarbonylvinylene)-metal.

\section{Introduction}

Russel (1948) has reported that Fries rearrangement (FR) of 4-acetoxybenzaldehyde gives the polymeric product containing chalcone groups in the polymer chains. Because of the good chelating properties of polymers prepared from 8-hydroxyquinoline (8 $\mathrm{HQ})$ (Pennington and Williams 1959; Vernon and Nyo 1978; Patel and Patel (1979); Patel et al 1984) and because of polymers based on the FR of 8-acetoxyquinoline-5-aldehyde (AQA) have not been reported so far, it was considered interesting to prepare such polymers to study their chelating properties. The work in the present communication is thus connected with the synthesis, characterization and chelation with metal ions of poly(8-hydroxy-5,7-quinolinylenecarbonylvinylene (PHQCV).

\section{Experimental}

\subsection{Materials and methods}

8-Hydroxyquinoline-5-aldehyde (HQA) was prepared by a method reported earlier (Sen and Ray 1932). 8-Acetoxyquinoline-5-aldehyde (AQA) was prepared by acetylation of HQA with excess of acetic anhydride in pyridine. All other chemicals used were of analar or chemically pure grade.

2.1a Preparation of polychalcone (PHQCV) by Fries rearrangement of AQA: To a well stirred and ice-cooled solution of $A Q A(0.1 \mathrm{~mol})$, finely powdered anhydrous aluminium trichloride $(0.2 \mathrm{~mol})$ in nitrobenzene $(6.0 \mathrm{ml})$ was added in small portions. The reaction mixture was allowed to come to room temperature and then heated to $100^{\circ} \mathrm{C}$ for $3 \mathrm{hr}$ and further to $120-130^{\circ} \mathrm{C}$ for $10 \mathrm{hr}$. The resultant dark green viscous reaction mixture was poured into ice cooled dilute $\mathrm{HCl}(500 \mathrm{ml}, 5 \%)$. Nitrobenzene was removed by steam distillation and the green solid was collected and air dried. It was Soxhletextracted with acetic acid to remove any unreacted monomer. The yield was $8.0 \mathrm{~g}$. 
2.1b Preparation of polymeric chelates of $\mathrm{PHQCV}$ polychalcone: Preparation of $\mathrm{PHQCV}-\mathrm{Cu}^{2+}$ chelate-To a solution of copper nitrate $(0.01 \mathrm{~mol})$ in $40 \mathrm{ml}$ of aqueous formic acid $(60 \% \mathrm{v} / \mathrm{v})$, a solution of PHQcv $(0.01 \mathrm{~mol})$ in $40 \mathrm{ml}$ of formic acid $(85 \% \mathrm{w} / \mathrm{v})$ at room temperature was added dropwise, with good stirring. The reaction mixture was then heated on a water-bath for $1 \mathrm{hr}$. The $\mathrm{pH}$ was adjusted to the required value $(4.5)$ by the addition of dilute ammonia solution when the polymeric chelate separated out in the form of a suspension. The latter was digested for $1 \mathrm{hr}$ on a steambath and collected by decantation. The polymeric chelate was filtered, washed with ethanol and then air dried. It was designated as $\mathrm{PHOCV}-\mathrm{Cu}^{2+}$

Following this method other metal chelates listed in table 1 were prepared.

\section{Analyses of PHQCv polychalcones and polymeric chelates}

(a) The nitrogen contents in the PHQCV polychalcone samples and the other polymeric chelates were estimated by Dumas' method. The analyses of metal ions in the polymeric chelates were carried out by the decomposition of a known amount of chelate by mineral acids. The metal content was estimated by a method reported in literature (Vogel 1979).

(b) The IR spectra of PHQCv polymer and polymeric metal chelates were scanned in $\mathrm{KBr}$ pellets on a Perkin-Elmer 983 Spectrophotometer.

(c) Conductometric titration of PHQcv polymer sample was carried out in pyridine against sodium methoxide $(\mathrm{NaOMe})$ in pyridine as standard. The value of the number average molecular weight $\left(\bar{M}_{n}\right)$ was calculated following a method of conductometric titration reported by Chatterjee (1971).

(d) Intrinsic viscosity [ $\eta]$ of the PHQCV polymer sample was estimated in $85: 15(\mathrm{v} / \mathrm{v})$ formic acid-acetic acid mixture at $30 \pm 0 \cdot 1^{\circ} \mathrm{C}$. Ubbelhode viscometer was used for this purpose.

(e) Thermogravimetry of the PHOCV polymer sample and all polymeric chelates was carried out on Linseis thermobalance at a heating rate of $10^{\circ} \mathrm{C} \mathrm{min}{ }^{-1}$.

\section{Results and discussions}

\subsection{Characterization of the polychalcone-PHQCV}

Fries rearrangement (FR) of 8-acetoxyquinoline-5-aldehyde (AQA) was carried out in nitrobenzene by varying temperature and the molar proportion of $\mathrm{AlCl}_{3}$. It was observed that the FR of AQA could not be effected below $100^{\circ} \mathrm{C}$ but only above $120^{\circ} \mathrm{C}$. It was also observed that more than $2 \mathrm{~mol}$ of $\mathrm{AlCl}_{3}$ yield a polymer which could not be isolated. This is because of the requirement of higher concentration of $\mathrm{HCl}$ for decomposition of more $\mathrm{AlCl}_{3}$ which simultaneously dissolved the polymeric product.

The PHQCV polymer sample was in the form of green powder, did not melt up to $360^{\circ} \mathrm{C}$ and was insoluble in common organic solvents. It was soluble only in formic acid and $\mathrm{HCl}$. Nitrogen content (table 1) of $\mathrm{PHQCV}$ polychalcone is consistent with the repeating units of the polymer chains. IR Spectrum of PHQCV polychalcone is shown in figure 1. The broad band appeared in the spectrum extending from 3600 to $2700 \mathrm{~cm}^{-1}$ belongs to chelated $\mathrm{OH}$ groups. Bands at 3030 and $3070 \mathrm{~cm}^{-1}$ have been assigned to the 


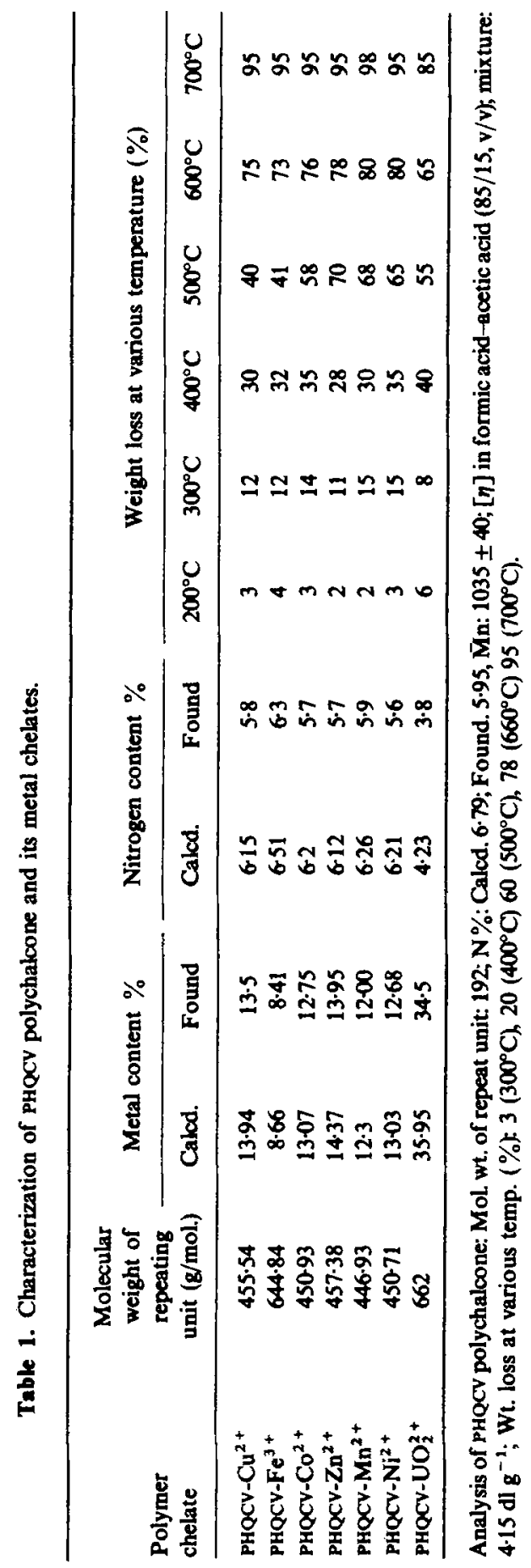




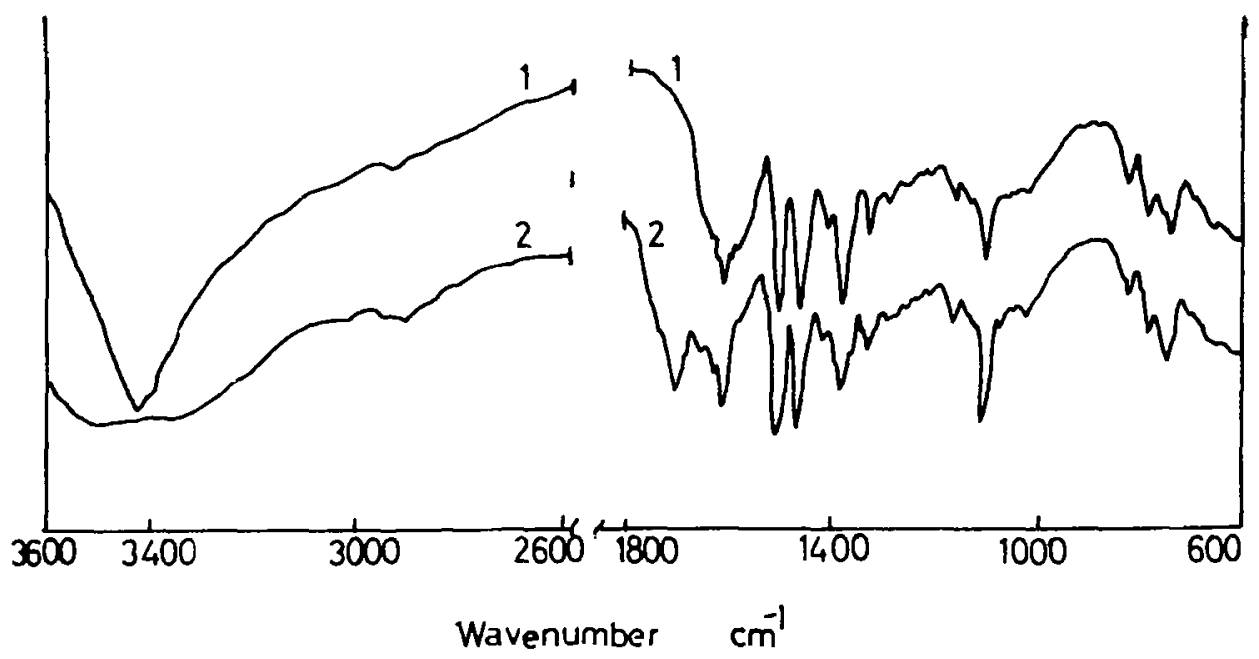

Figure 1. IR Spectra of $1 \mathrm{PHQCV}$ polychalcone; $2 \mathrm{PHQCV}-\mathrm{Cu}^{2+}$ polychelate.

aromatic $\mathrm{C}-\mathrm{H}$ stretching vibration. In this region no separate band is observed due to $v_{\mathrm{CH}}$ of chalcone group. A broad band around $1680 \mathrm{~cm}^{-1}$ may be attributed to $v_{\mathrm{C}=0}$ of conjugated $-\stackrel{O}{\mathrm{C}}-\mathrm{CH}=\mathrm{CH}$ system (Mecke and Noack 1960). The strong bands around 1300 and $1200 \mathrm{~cm}^{-1}$ due to $v_{\mathrm{C}-\mathrm{N}}$ are also observed. However the band due to conjugated $-\mathrm{C}=\mathrm{C}-$ could not be discerned distinctly. Hence this was confirmed by bromination of PHQCV polymer. The bromine content of brominated PHQCV consist with the presence of chalcone group in the parent polymer chain.

These suggest that the FR of AQA may be postulated by the formation of the ketoaldehyde intermediate and its spontaneous self-polycondensation via Aldol condensation in an acidic medium to polychalcone PHQcv by the route shown in chart 1.

The number average molecular weight $\left(\bar{M}_{n}\right)$ (table 1) of PHQCv polychalcone sample determined by conductometric titration was found to be 1035 . The intrinsic viscosity

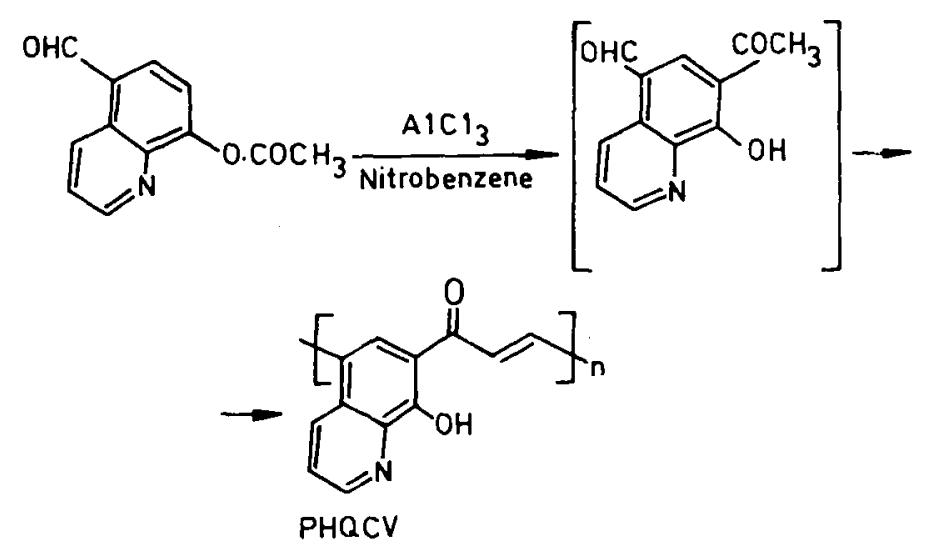

Chart 1. 
[ $\eta]$ of PAHQ polychalcone in formic acid-acetic acid $(85: 15, \mathrm{v} / \mathrm{v})$ mixture was found to be $4.15 \mathrm{dl} \mathrm{g}^{-1}$. The results of TG analysis of PHQCV polychalcone show that it starts decomposition at a perceptible rate at some temperature around $300^{\circ} \mathrm{C}$. Beyond this temperature the polymer samples degrade very rapidly and loses its weight completely round $700^{\circ} \mathrm{C}$. Comparison of the thermal properties of the produced polychalcone with those of polymers derived from $8 \mathrm{HQ}$ (Pennington and Williams 1959; Patel and Patel 1979; Patel et al 1984) reveals that PHQCV is less stable. This may be due to thermooxidation reactions in the polymer chain which arise due to the dehydrogenation of OH groups ( Jackson and Conley 1964) and the formation of the new carbonyl groups of their acidic or quinoid type radicals (chart 2).

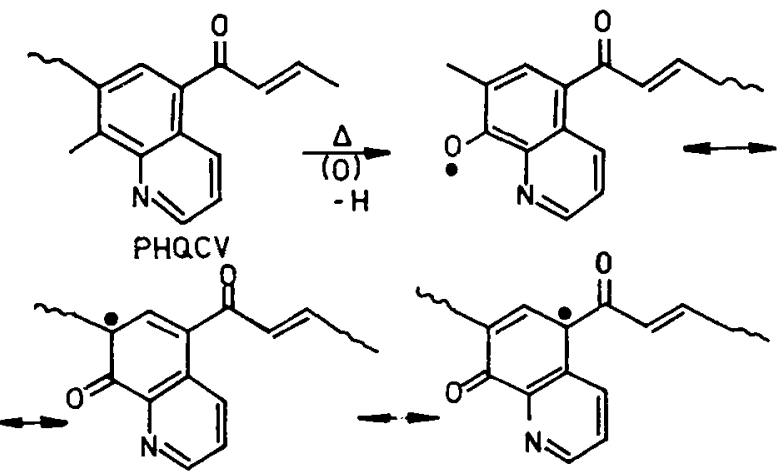

Chart 2.

\subsection{Characterization of chelates of PHQCV}

All the polymeric chelates presented in table 1 are found to be insoluble in common organic solvents. They did not melt up to $360^{\circ} \mathrm{C}$. The results of metal and nitrogen analyses are shown in table 1 . The values for the metal contents suggested that the metal : ligand ratio is $1: 2$ for all bivalent metal ions and $1: 3$ for $\mathrm{Fe}^{3+}$ ions. These results were also supported by nitrogen analyses of polymeric chelates.

The IR spectra of the polymeric chelates resembled each other in their general shape and in the relative intensity of bands. Comparison of the IR spectrum of the polymeric ligand and of the polymeric chelate $\mathrm{PHQCV}-\mathrm{Cu}^{2+}$ (figure 1) reveals that the broad absorption in the region $3500-2500 \mathrm{~cm}^{-1}$ in the spectrum of the polymeric ligand, due to chelated $\mathrm{OH}$ groups, are much less broad in the spectrum of the polymeric chelate. This indicates the absence of internal H-bonding due to chelation, which is in agreement with the studies of coordination polymers of bis(oxine) (Horowitz and Perros 1964). In the IR spectra of polymeric chelates the band around $1700 \mathrm{~cm}^{-1}$ due to $v_{\mathrm{C}-\mathrm{O}}$ appeared more clearly. Hence, it can be inferred that the carbonyl of the chalcone group could not participate in the chelation. The weak band appearing at $1100 \mathrm{~cm}^{-1}$ is attributed to the $\mathrm{C}-\mathrm{O}-M$ stretching frequency (Charles et al 1958). All these features suggest the structure of polychelates is as in chart 3.

Examination of the thermograms and thermal analysis (table 2) of all the polymeric chelates reveales that like $P H Q C V$ polychalcone, each polymeric chelate degrade above $300^{\circ} \mathrm{C}$. The rate of decomposition of the polymeric chelate is comparatively thermally less stable than PHQCV. It seems that the metal ions accelerate the decomposition of the polymeric chelate (Horowitz and Perros 1964). 


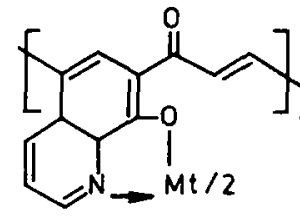

$$
\begin{gathered}
\mathrm{Mt}: \mathrm{Cu}^{2+}, \mathrm{Ni}^{2+} \cdot \mathrm{CO}^{2+} \cdot \\
\mathrm{Zn}^{2+}, \mathrm{Mn}^{2+}, \mathrm{UO}_{2}^{2+}
\end{gathered}
$$

$\mathrm{Mt} / 3: \mathrm{Fe}^{3+}$

Chart 3.

\section{Acknowledgements}

The author is thankful to the Milton Group, Bombay, for TGA. The author expresses his gratitude to Prof. R P Patel for encouragement.

\section{References}

Charles R G, Freiser H, Friedel R, Milliand L F and Johnston R D 1958 Spectrochim. Acta 81

Chatterjee S K 1970 J. Polym. Sci. A8 1299

Chatterjee S K $1971 \mathrm{~J}$. Polym. Sci. A9 3225

Horrowitz H and Perros J P 1964 J. Inorg. Nucl. Chem. 26139

Jackson W M and Conley R T 1964 J. Appl. Polym. Sci. 82873

Meck R and Noack K 1960 Chem. Ber. 93210

Patel A U, Patel H S and Patel M N 1984 Angew. Makromol. Chem. 131135

Patel H S and Patel S R 1979 Makromol. Chem. 180 887, 897, 1159

Pennington L D and Williams M B 1959 Ind. Eng. Chem. 51759

Russel A 1948 J. Am. Chem. Soc. 702864

Sen R N and Ray S K 1932 J. Indian Chem. Soc. 9173

Vernon F and Nyo L M 1978 J. Inorg. Nucl. Chem. 40887

Vogel A I 1979 A text book of practical inorganic chemistry 4th ed. (New York: Longmans) 